\title{
Iris Melanoma
}

National Cancer Institute

\section{Source}

National Cancer Institute. Iris Melanoma. NCI Thesaurus. Code C9088.

A uveal melanoma that arises from the iris. It is the most common primary malignant neoplasm of the iris. The majority arise in preexisting nevi. 